\title{
Die Rolle der Selbstbestimmung in der Rechtfertigung passiver und aktiver Sterbehilfe
}

von Andreas Müller

\begin{abstract}
Wann und auf welche Weise dürfen Ärzte am Tod eines Patienten mitwirken? Der vorliegende Beitrag widmet sich der argumentativen Rolle, die die Selbstbestimmung des Patienten in der Auseinandersetzung über diese Frage spielt. Auf der Grundlage einer detaillierten Untersuchung der moralischen Relevanz von Einwilligungen als einer wesentlichen Ausprägung dieser Selbstbestimmung wird zum einen dafür argumentiert, dass es einen moralisch signifikanten Unterschied zwischen passiver - d. h. mit dem Verzicht auf eine lebensverlängernde medizinische Maßnahme einhergehender - und aktiver Sterbehilfe gibt. Die Zulässigkeit der aktiven Sterbehilfe kann folglich nicht schon mittels eines Äquivalenzarguments aus der Zulässigkeit der passiven abgeleitet werden. Zum anderen aber lässt sich auf derselben Grundlage zeigen, wie ein am Selbstbestimmungsrecht des Patienten ansetzendes Argument für die Zulässigkeit mancher Fälle von aktiver Sterbehilfe präzisiert und gegen zwei verbreitete Einwände verteidigt werden kann.
\end{abstract}

Keywords: passive Sterbehilfe, aktive Sterbehilfe, Selbstbestimmung, Einwilligung, Äquivalenzargument

\section{Zur Debattenlage}

Wann und auf welche Weise dürfen Ärzte am Tod eines Patienten mitwirken? In der Auseinandersetzung über diese Frage wird oft zwischen verschiedenen Arten von Fällen unterschieden, für welche die beiden folgenden paradigmatische Beispiele sind:

Fall 1: Ein sterbenskranker Patient leidet in aus seiner Sicht unerträglichem Maße an starken Schmerzen und den Einschränkungen der Kommunikations- und Bewegungsfähigkeit, die seine Erkrankung unweigerlich mit sich bringt. Er erklärt darum, dass er nicht wiederbelebt werden möchte, sollte sein Herz-Kreislauf-System versagen. Es kommt zu einem solchen Versagen und sein Arzt unternimmt nichts, um das Herz des Patienten wieder zum Schlagen zu bringen, woraufhin dieser verstirbt.

Fall 2: Ein sterbenskranker Patient leidet in aus seiner Sicht unerträglichem Maße an starken Schmerzen und den Einschränkungen der Kommunikations- und Bewe- 
gungsfähigkeit, die seine Erkrankung unweigerlich mit sich bringt. Er bittet darum seinen Arzt, sein Leben auf möglichst rasche und schmerzfreie Weise zu beenden. Der Arzt kommt dieser Bitte schließlich nach und verabreicht dem Patienten eine tödliche Injektion, woraufhin dieser verstirbt.

In beiden Fällen handelt es sich um ärztliche Sterbehilfe. Darunter sollen hier alle Vorgänge verstanden werden, in denen ein Arzt am Herbeiführen des Todes eines Patienten beteiligt ist, ${ }^{1}$ dies dem Willen des Patienten entspricht und der Arzt in Kenntnis dieses Patientenwillens sowie der (voraussichtlich) tödlichen Folgen für den Patienten handelt. Ein Herbeiführen des Todes liegt dann vor, wenn der Zeitpunkt des Todes des Patienten so beeinflusst wird, dass dieser signifikant früher eintritt, als andernfalls zu erwarten gewesen wäre. Natürlich können nicht nur Ärzte, sondern etwa auch Angehörige Sterbehilfe leisten. Dennoch beschränke ich mich hier auf die Diskussion ärztlicher Sterbehilfe, da in den meisten Fällen bei der Beurteilung der Situation, der Aufklärung des Patienten sowie der Mitwirkung am Herbeiführen des Todes medizinische Fachkenntnisse erforderlich sind und Ärzte folglich mit der Entscheidung für oder gegen ein solches Mitwirken besonders häufig konfrontiert sind. Zudem wirft der Umstand, dass eine bestimmte Handlung durch einen Arzt ausgeführt wird, zusätzliche Fragen auf, die, wie wir noch sehen werden, im Kontext der Sterbehilfe besonders relevant sind.

Der Unterschied zwischen den beiden genannten Fällen wird insbesondere in der öffentlichen Diskussion häufig mit dem Begriffspaar aktiv/passiv in Verbindung gebracht. In Fall 1 leistet der Arzt demzufolge passive Sterbehilfe, während es sich bei Fall 2 um aktive Sterbehilfe handle. Über die prinzipielle Zulässigkeit der ersten Form von Sterbehilfe besteht dabei breites Einvernehmen. Kontroverser ist dagegen die moralische Bewertung von aktiver Sterbehilfe, wie sie Fall 2 illustriert. Vertreter einer restriktiven Position halten lediglich die passive Sterbehilfe für zulässig, lehnen aktive Sterbehilfe aber als grundsätzlich moralisch unzulässig ab. Andere beziehen eine liberale Position, der zufolge auch aktive Sterbehilfe in bestimmten Situationen moralisch zulässig sein kann. ${ }^{2}$

1 „Beteiligung“ soll hier zunächst so weit verstanden werden, dass auch der Arzt an der Herbeiführung des Todes eines Patienten beteiligt ist, der ein Mittel (beispielsweise eine tödliche Dosis eines Betäubungsmittels) bereitstellt, mit dessen Hilfe sich der Patient selbst tötet. Ärztliche Suizidbeihilfe gilt demnach als eine Form von Sterbehilfe. Davon ist unbenommen, dass die Art oder der Grad der Beteiligung des Arztes - d. h. das, worin sich das Bereitstellen eines tödlichen Medikaments vom Injizieren eines solchen Medikamentes unterscheidet - einen Unterschied für die moralische Bewertung seines Handelns machen kann. Ob dies so ist, wird im Folgenden jedoch nicht eigens untersucht.

2 Beide hier genannten Positionen betreffen die moralische Zulässigkeit von Sterbehilfe, nicht die Frage, ob diese rechtlich erlaubt oder verboten werden sollte. Die moralische Bewertung von Handlungsweisen ist zwar gewiss nicht irrelevant für deren rechtliche 
Grundsätzlich gilt, dass wenn zwei Handlungen sich in ihrem moralischen Status unterscheiden, auch ein moralisch relevanter Unterschied zwischen diesen Handlungen bestehen muss. Handlungen, die sich in keiner moralisch relevanten Hinsicht unterscheiden, müssen auch hinsichtlich ihrer moralischen Zulässigkeit gleich beurteilt werden. Die restriktive Position, die ja die Zulässigkeit passiver Sterbehilfe nicht bestreitet, setzt somit voraus, dass die Unterscheidung zwischen aktiver und passiver Sterbehilfe einen moralisch relevanten Unterschied markiert. Daraus ergibt sich, dass eine Möglichkeit zur Begründung der liberalen Position darin besteht zu zeigen, dass zumindest in bestimmten Situationen kein moralisch relevanter Unterschied zwischen aktiver und passiver Sterbehilfe besteht. Lässt sich diese Äquivalenzthese verteidigen, so kann aus ihr, zusammen mit der Zulässigkeit passiver Sterbehilfe, angesichts des soeben erläuterten Zusammenhanges gefolgert werden, dass auch aktive Sterbehilfe in bestimmten Situationen zulässig ist. Dieses Äquivalenzargument für die Zulässigkeit aktiver Sterbehilfe hat einen festen Stand in der gegenwärtigen Debatte. ${ }^{3}$

Das Äquivalenzargument ist aber natürlich nicht die einzige Option für die Begründung einer liberalen Position. Alternativ kann man auch direkt für die Zulässigkeit aktiver Sterbehilfe zu argumentieren versuchen. Eine solche Argumentationsweise rekurriert in ihren Prämissen nicht auf die Zulässigkeit passiver Sterbehilfe; sie benennt vielmehr die für und gegen die aktive Sterbehilfe sprechenden Gesichtspunkte und beansprucht zu zeigen, dass erstere letztere in manchen Situationen überwiegen. Dabei wird meist in der einen oder anderen Weise auf das Selbstbestimmungsrecht bzw. die Autonomie des Patienten Bezug genommen. ${ }^{4}$ Aktive Sterbehilfe ist demzufolge zulässig, wenn und insoweit sie dem (autonomen) Patientenwillen entspricht, den es auch im Falle von Entscheidungen über den eigenen Tod zu respektieren gelte. Eine solche Argumentation kann zugestehen, dass es

Regelung. Es ist aber weder inkonsistent, sich gegen ein rechtliches Verbot von moralisch unzulässigen Handlungen (z. B. Vertrauensbrüchen) auszusprechen, noch für ein rechtliches Verbot von Handlungsweisen zu plädieren, die man in bestimmten Fällen für moralisch zulässig hält (etwa weil andernfalls damit zu rechnen wäre, dass solche Handlungen vielfach auch in Situationen ausgeführt würden, in denen sie nicht zulässig sind). Im Folgenden geht es allein um die moralische Bewertung verschiedener Formen von Sterbehilfe.

3 Vgl. etwa Wolf (1993, 414), RaChels (1989, 261 ff.), BirnbaCHer (1995, 348 ff.), SCHÖNE-SEIFERT (1997, 217 ff.) und SuMNER (2011, 23). Grundsätzlich erlaubt die Äquivalenzthese natürlich auch eine Argumentation in entgegengesetzter Richtung, d. h. von der Unzulässigkeit aktiver für die Unzulässigkeit passiver Sterbehilfe. Angesichts des Umstandes, dass der philosophische wie auch der gesellschaftliche Dissens die Zulässigkeit der aktiven und gerade nicht die der passiven Sterbehilfe betrifft, wird diese Option, der es zudem in der gegenwärtigen Diskussion an ernst zu nehmenden Vertretern mangelt, hier jedoch nicht weiter berücksichtigt.

4 Vgl. Dworkin (1993, Kap. 7), Quante (1998 sowie 2002, Kap. 6) und GutmanN (2002). 
einen moralisch relevanten Unterschied zwischen der aktiven und der passiven Sterbehilfe gibt, bestreitet jedoch, dass dieser stets den Ausschlag gegen die Zulässigkeit aktiver Sterbehilfe gibt.

Hier zeigt sich, dass das Bestreiten der Äquivalenzthese zwar notwendig, aber allein nicht hinreichend für die Begründung einer restriktiven Position ist. Zunächst muss der moralisch relevante Unterschied gewissermaßen auch in die richtige Richtung weisen, d. h. es muss gelten, dass aktive Sterbehilfe aufgrund dieses Unterschiedes moralisch problematischer als passive Sterbehilfe ist, oder dass zur Rechtfertigung der letzteren mehr argumentative Ressourcen zur Verfügung stehen als für erstere. Vor allem aber müssen zwei Handlungen, die sich in solcher Weise unterscheiden, sich deshalb nicht auch hinsichtlich ihrer moralischen Zulässigkeit unterscheiden. In beiden Fällen kann schließlich so viel für die Handlung sprechen, dass es die problematischen Gesichtspunkte überwiegt und die Handlung folglich zulässig ist, obwohl die gegen oder für die Handlung sprechenden Gesichtspunkte im einen Fall gewichtiger bzw. weniger gewichtig sind als im anderen. Der Vertreter einer restriktiven Position ist also nicht nur darauf festgelegt, dass es einen moralisch relevanten Unterschied zwischen aktiver und passiver Sterbehilfe gibt, sondern auch darauf, dass dieser moralisch entscheidend ist, dass also anders als im Falle der passiven Sterbehilfe bei der aktiven Sterbehilfe die moralisch problematischen Gesichtspunkte nicht durch die zur Verfügung stehenden Rechtfertigungsressourcen aufgewogen werden können.

Diese Darstellung der dialektischen Situation in der Diskussion über die Zulässigkeit aktiver Sterbehilfe macht deutlich, dass die Frage, ob die Unterscheidung zwischen aktiver und passiver Sterbehilfe einen moralisch relevanten Unterschied markiert, sowohl für Vertreter einer liberalen als auch für Vertreter einer restriktiven Position von zentraler Bedeutung ist. Für erstere würde eine negative Antwort die entscheidende Prämisse des Äquivalenzargumentes für die Zulässigkeit der aktiven Sterbehilfe bereitstellen, während für letztere eine positive Antwort als erster Schritt für die Begründung der Unzulässigkeit aktiver Sterbehilfe erforderlich ist. Die Beantwortung der Frage wird jedoch dadurch erschwert, dass die aktiv/passiv-Unterscheidung in der öffentlichen Diskussion sowie in den verschiedenen Fachdebatten ganz unterschiedlich verstanden wurde und wird und somit die Gefahr besteht, dass die Unterscheidung, die der eine für moralisch irrelevant hält, gar nicht die Unterscheidung ist, die der andere zur Begründung seiner Position heranzuziehen gedenkt.

Vor diesem Hintergrund gehe ich im vorliegenden Beitrag der Frage nach, ob und wie sich die These der Unzulässigkeit aktiver Sterbehilfe angesichts des Konsenses über die Zulässigkeit passiver Sterbehilfe begründen lässt. Dafür stelle ich zunächst die gegenwärtig verbreitetste und überzeugendste Explikation der aktiv/passiv-Unterscheidung vor und grenze sie von anderen, gelegentlich noch immer für Verwirrung sorgenden Verständnisweisen ab. Anschließend wird untersucht, inwiefern die so verstandene Unterscheidung einen moralisch relevanten Unterschied markiert. Hierfür ist eine detaillierte Diskussion der Rolle, die die Einwilligung des Patienten in der Rechtfertigung aktiver und passiver Sterbehilfe spielt, 
sowie des Verhältnisses solcher Einwilligungen zu anderen Elementen der Selbstbestimmung des Patienten erforderlich. Die Ergebnisse dieser Diskussion zeigen zum einen, dass durchaus ein Unterschied in den zur Verfügung stehenden Rechtfertigungsressourcen für aktive und passive Sterbehilfe besteht, sodass die Äquivalenzthese als zentrale Prämisse des Äquivalenzargumentes zurückgewiesen werden muss. Zum anderen aber machen diese Ergebnisse deutlich, dass Vertreter der restriktiven Position ein direktes Argument für die Zulässigkeit aktiver Sterbehilfe, das an der Einwilligung als Ausprägung der Selbstbestimmung des Patienten ansetzt, nur durch Rekurs auf nicht plausibel begründbare Thesen zurückweisen können. Schließlich zeige ich, dass ein angemessenes Verständnis der Rolle von Selbstbestimmung es außerdem ermöglicht, zwei verbreitete Einwände gegen ein solches direktes Argument zurückzuweisen.

\section{Sterbehilfe und Behandlungsverzicht}

In der medizinethischen und rechtlichen Fachdiskussion ist es üblich geworden, die verschiedenen Arten von Sterbehilfe, für die Fall 1 und Fall 2 jeweils paradigmatisch sind, danach zu unterscheiden, ob sie mit dem Verzicht auf eine medizinische Behandlung verbunden sind, die für den Patienten lebensnotwendig bzw. lebensverlängernd wäre. ${ }^{5}$ Von einigen ist diese Unterscheidung anhand des Behandlungsbezugs als ein Ersatz für die als irreführend oder zu unklar wahrgenommene aktiv/passiv-Unterscheidung vorgeschlagen worden. ${ }^{6}$ Angesichts der fest verankerten Position, die letztere Unterscheidung insbesondere in der öffentlichen Debatte hat, erscheint es jedoch sinnvoller, nicht zu versuchen, die etablierten Begrifflichkeiten abzuschaffen und durch andere zu ersetzen, sondern das Kriterium des Behandlungsbezuges als eine Explikation der aktiv/passiv-Unterscheidung zu betrachten und an der Rede von aktiver und passiver Sterbehilfe schon aus pragmatischen Gründen festzuhalten. Passive Sterbehilfe besteht danach im Herbeiführen des Todes durch den Verzicht auf oder den Abbruch einer lebenserhaltenden bzw. -verlängernden medizinischen Maßnahme. ${ }^{7}$ Aktive Sterbehilfe dagegen liegt

5 Vgl. exemplarisch SCHÖNE-SEIFERT $(2007,114)$ und ACH ET AL. (2012, 234) sowie NATiOnALER Ethikrat (2006, 54), Bundesgerichtshof (2010, Rn. 31) und BUNDESÄRZTEKAMMER (2011a, A346).

6 Vgl. etwa Nationaler Ethikrat $(2006,54)$ und Bundesgerichtshof (2010, Rn. 31).

7 Die Rede von „Maßnahmen“ anstelle von „Behandlungen“ erlaubt es, Komplikationen zu vermeiden, die sich zum einen daraus ergeben, dass eine Behandlung einen behandlungsbedürftigen Zustand vorauszusetzen scheint und darum in Frage gestellt werden kann, ob es sich beispielsweise beim Einstellen einer künstlichen Ernährung um einen Behandlungsabbruch handelt, vgl. HöFLING/RiXEN (2003, 888 f.). Zum anderen kann 
vor, wenn der Tod des Patienten in einer Weise herbeigeführt wird, die keinen solchen Bezug zu einer lebenserhaltenden Maßnahme hat.

Diese Explikation der Unterscheidung zwischen aktiver und passiver Sterbehilfe ist von anderen Differenzierungsversuchen abzugrenzen, die ebenfalls mit der aktiv/passiv-Unterscheidung in Verbindung gebracht werden. Dies ist zum einen die Unterscheidung zwischen Tun und Unterlassen als verschiedenen performativen Modi menschlichen Handelns im weiteren Sinne. ${ }^{8}$ Fälle wie der folgende machen klar, dass diese sich nicht mit der Unterscheidung zwischen aktiver und passiver Sterbehilfe deckt:

Fall 3: Ein Arzt schaltet in Übereinstimmung mit dem Willen eines schwerstkranken Patienten dessen Beatmungsmaschine aus, woraufhin dieser verstirbt.?

Hier wird der Tod des Patienten durch ärztliches Tun herbeigeführt. Dennoch werden solche Fälle gemeinhin - und in Übereinstimmung mit der oben vorgestellten Explikation - als passive Sterbehilfe klassifiziert.

Auch der Kontrast zwischen Formen der Sterbehilfe, bei denen es sich um eine Tötung des Patienten handelt, und solchen, für die dies nicht gilt, ist bei der Explikation der Unterscheidung zwischen aktiver und passiver Sterbehilfe wenig hilfreich. ${ }^{10}$ Versteht man diesen Kontrast so, dass eine Person nur dann eine andere tötet, wenn sie dadurch eine moralische Pflicht verletzt und also zumindest prima facie moralisch unzulässig handelt, ${ }^{11}$ dann eignet er sich nicht, um eine unterschiedliche moralische Bewertung der entsprechenden Handlungen zirkelfrei zu begründen. Meint man dagegen, dass nur derjenige einen anderen tötet, der den Tod dieser Person (mit-)verursacht, ${ }^{12}$ läge auch in Fall 3 eine Tötung vor. ${ }^{13}$

auch der Verzicht auf eine für die erfolgreiche Durchführung einer lebensverlängernden Behandlung erforderliche diagnostische Maßnahme den Tod des Patienten herbeiführen.

8 Zu dieser Unterscheidung und ihrer Relevanz im Kontext der Sterbehilfe-Problematik vgl. ausführlich BIRNBACHER (1995).

9 Wie die Fälle 1 und 2 dient auch dieser Fall dazu, spezifische, für den Argumentationsgang zentrale Aspekte verschiedener Arten der Sterbehilfe herauszustellen. Dies macht es erforderlich, von zahlreichen anderen Aspekten zu abstrahieren, wodurch die Beschreibungen dieser Fälle an Nähe zu konkreten Situationen einbüßen. Gleichwohl sind viele reale Situationen ihnen in den relevanten Hinsichten ähnlich.

10 „Töten“ wird in diesem Zusammenhang oft mit „Sterbenlassen“ kontrastiert. Der Aspekt des bloßen Geschehenlassens des Todes zeichnet in der Tat die passive Sterbehilfe aus; seine Relevanz wird unten in Abschnitt 3 diskutiert.

11 So etwa GERT ET AL. (2006, 322 f.), die allerdings auch nicht vorschlagen, die aktiv/passiv-Unterscheidung mit der zwischen Töten und Sterbenlassen zu identifizieren.

12 So etwa Brody (1989, 302), vgl. auch RACHELs (1989, 261 f.). 
Eine weitere Unterscheidung, die in der Diskussion über die Zulässigkeit verschiedener Formen von Sterbehilfe eine Rolle spielt, betrifft die mentalen Zustände des Handelnden: Beabsichtigt dieser, den Tod des Patienten herbeizuführen, oder nimmt er dessen Tod lediglich als unbeabsichtigte, aber nicht vermeidbare Nebenfolge seines Handelns in Kauf? Hier ist oft von direkter bzw. indirekter Sterbehilfe die Rede. Diese Unterscheidung steht quer zu der zwischen aktiver und passiver Sterbehilfe, denn der Verzicht auf eine intensivmedizinische Behandlung kann ebenso wie das Verabreichen eines medizinisch indizierten Schmerzmittels sowohl mit der Absicht erfolgen, Leid zu mindern, als auch mit der, den Tod zu beschleunigen. Als Explikation des durch Fall 1 und Fall 2 illustrierten Unterschiedes zwischen aktiver und passiver Sterbehilfe eignet sich die Unterscheidung zwischen Beabsichtigen und Inkaufnehmen folglich nicht.

Manche halten diese Unterscheidung allerdings für die eigentlich moralisch relevante und beurteilen daher nur direkte aktive Sterbehilfe als unzulässig. Zur Begründung der unterschiedlichen Bewertung von Beabsichtigen und Inkaufnehmen des Todes berufen sie sich dabei auf die Doppeleffekt-Doktrin. Dieser zufolge hängt die Zulässigkeit einer Handlung mit sowohl guten als auch schlechten Folgen davon ab, ob der Handelnde die schlechten Folgen (als Zweck oder Mittel) zu realisieren beabsichtigt oder sie nur als unvermeidbaren Nebeneffekt der Realisierung der guten Folgen in Kauf nimmt. Auch abgesehen von grundsätzlichen Zweifeln an der Plausibilität dieser Doktrin ${ }^{14}$ ist jedoch fraglich, inwiefern sie in den zur Diskussion stehenden Fällen überhaupt angewendet werden kann. Schließlich würde sie damit, dass es sich beim Tod des Patienten auch dann um eine schlechte Folge handelt, wenn dieser der Vermeidung von großem Leid dient und dem Patientenwillen entspricht, etwas voraussetzen, was viele Befürworter einer liberalen Position bestreiten. ${ }^{15}$ Angesichts dieses und weiterer Probleme eines solchen Differenzierungsversuchs ${ }^{16}$ steht hier deshalb die Unterscheidung zwischen aktiver und passiver Sterbehilfe im eingangs explizierten Verständnis sowie die Frage nach ihrer moralischen Relevanz im Mittelpunkt.

13 Zudem erlaubt es dieser Vorschlag u. U. nicht einmal, zwischen Fall 1 und Fall 2 zu differenzieren, denn einer plausiblen Kausalitätskonzeption zufolge können auch Unterlassungen ein Ereignis (mit-)verursachen, vgl. BIRNBACHER (1995, Kap. 3).

14 Einen aktuellen Überblick über die Diskussion bietet MCINTYRE (2014).

15 Dies gilt beispielsweise für Anhänger einer Konzeption des Unwertes des Todes, wonach dieser nur insoweit schlecht ist, als das durch ihn verhinderte weitere Leben selbst ein Gut darstellt, letzteres jedoch nicht immer der Fall ist. Vgl. dazu exemplarisch Williams (1973), WitTWER (2003, 241 ff.) und SumnER (2011, 11 ff.).

16 Vgl. die kritischen Diskussionen bei Birnbacher (1995, 153 ff. und 345 f.), SCHÖNESEIFERT (1997, 220 ff.), QuANTE (2002, 248 f.) sowie SumnER (2011, Kap. 3). 


\section{Ist die Unterscheidung moralisch relevant?}

Ist die so verstandene Unterscheidung zwischen passiver und aktiver Sterbehilfe moralisch signifikant? Bringt die Unterscheidung einen Unterschied in den Rechtfertigungsressourcen mit sich, die für passive und aktive Sterbehilfe zur Verfügung stehen? Die Antwort auf diese Frage ist einigermaßen kontrovers. Das liegt unter anderem daran, dass man zwei verschiedene Aspekte der medizinischen Maßnahme, auf die im Rahmen passiver Sterbehilfe verzichtet werden soll, in den Vordergrund stellen und auf ihre moralische Relevanz hin untersuchen kann, nämlich einerseits, was die Maßnahme bewirken würde, und andererseits, wie sie es bewirken würde.

Angesichts der Explikation von passiver Sterbehilfe als Verzicht auf lebensverlängernde Maßnahmen (oder als deren Abbruch) liegt es nahe, den Blick zunächst auf den erstgenannten Aspekt und insbesondere auf den folgenden Umstand und dessen Rechtfertigungspotential zu richten: Bei der passiven Sterbehilfe wird der Tod des Patienten wesentlich (wenn auch nicht allein) durch einen unabhängigen Prozess verursacht, der bereits vor Beginn der Maßnahme seinen Anfang genommen hat. Dieser könnte durch die Maßnahme allerdings aufgehalten oder zumindest verzögert werden. Wer passive Sterbehilfe leistet, lässt somit lediglich einem unabhängigen Prozess seinen Lauf, d. h. er lässt etwas geschehen, wobei ein solches Geschehenlassen sowohl durch Unterlassen (z. B. einer Wiederbelebung) als auch durch Tun (z. B. Ausschalten einer Beatmungsmaschine) erfolgen kann. ${ }^{17}$ Im Gegensatz dazu wird bei der aktiven Sterbehilfe der zum Tod des Patienten führende Prozess erst in Gang gesetzt - hier stirbt der Patient somit nicht in Folge eines unabhängigen oder, wie es dann häufig heißt, nicht in Folge eines natürlichen Sterbeprozesses. Macht dies einen moralischen Unterschied? Hier ist Skepsis angebracht.

Erstens ist fraglich, ob der Verweis auf die vermeintliche Natürlichkeit des Todes bei der passiven im Gegensatz zur aktiven Sterbehilfe etwas auszutragen vermag. Zunächst ist alles andere als klar, unter welchen Bedingungen der Prozess, der zum Tod eines Patienten führt, als ein „,natürlicher“ gelten kann, und ob dies bei passiver Sterbehilfe stets der Fall ist: Inwiefern ist beispielsweise der durch ein ärztlich verabreichtes oder bereitgestelltes Betäubungsmittel herbeigeführte Tod weniger natürlich als der eines durch einen Sportunfall halsabwärts Gelähmten, bei dem die Beatmungsmaschine abgeschaltet wird? Zwar wird - in einem aus Krimis und ähnlichen Kontexten bekannten Sinne - etwa beim Ausfüllen eines Totenscheins zwischen einem natürlichen und einem nicht-natürlichen Tod unterschieden. Dabei handelt es sich jedoch um eine forensische, d.h. mit einem bestimmten Erkenntnisinteresse getroffene Unterscheidung, die darauf abzielt, einen Suizid oder ein Fremdverschulden des Todes auszuschließen. Sie klassifiziert darum auch den Tod infolge des Abbruchs einer künstlichen Beatmung als „nicht natürlich“, wenn die

17 Vgl. Birnbacher (1995, 110 ff.) und GeSANG (2001). 
Beatmung beispielsweise durch eine bei einer Schlägerei erlittene Hirnverletzung erforderlich geworden ist. Zudem und vor allem aber ist nicht ersichtlich, warum und inwiefern es besser oder weniger problematisch sein sollte, wenn jemand infolge eines natürlichen Prozesses stirbt. „Die“ Natur ist weder mitfühlend noch wohlwollend oder gnädig, und natürliche Prozesse werden oft als ein Übel empfunden, das wir gerade auch mit den Mitteln der Medizin aufzuhalten versuchen. ${ }^{18}$

Zweitens macht der Umstand, dass ein Tod Folge eines unabhängigen, nicht-aufgehaltenen Prozesses und nicht eines durch den Arzt angestoßenen Prozesses ist, zumindest in manchen Kontexten keinen Unterschied für die moralische Bewertung. Dies illustrieren die folgenden zwei Fälle. Im ersten versäumt es ein Arzt, die Infektion eines Patienten rechtzeitig zu diagnostizieren, weil er die Ergebnisse eines Bluttests in der Krankenakte übersieht, so dass der Patient an den Folgen der Infektion stirbt. Im zweiten Fall infiziert der Arzt einen Patienten versehentlich mit einem Erreger, weil er sich vor der Untersuchung die Hände nicht desinfiziert, und auch hier verläuft die Infektion tödlich. Es erscheint mir nicht plausibel, hier moralisch differenzieren zu wollen. In beiden Fällen verletzt der Arzt seine Sorgfaltspflicht, und in beiden Fällen kommt es so zu einem Todesfall, den der Arzt leicht hätte vermeiden können. Aber nur im ersten Fall kann der Tod auf einen unabhängigen Prozess zurückgeführt werden, den der Arzt lediglich nicht aufhält. Dieser Umstand ist hier also anscheinend moralisch irrelevant. Daraus folgt zwar nicht zwingend, dass dieser Unterschied auch in anderen Kontexten moralisch nicht signifikant ist. ${ }^{19}$ Es zeigt aber, dass sich die These, im Falle der Sterbehilfe sei an dieser Stelle moralisch zu differenzieren, nur aufrechterhalten lässt, wenn zugleich begründet werden kann, warum der Unterschied im Falle von Sterbehilfe eine unterschiedliche moralische Bewertung rechtfertigen sollte, in den genannten Fällen versehentlichen tödlichen Handelns aber nicht. Es ist meines Erachtens nicht zu sehen, wie eine solche Begründung aussehen könnte. ${ }^{20}$

Die verbreitete Skepsis bezüglich der moralischen Relevanz des Umstandes, dass der Tod eines Patienten durch das Geschehenlassen eines unabhängigen Prozesses herbeigeführt wird, erscheint mir daher berechtigt. Folgt daraus, dass die Unter-

18 Vgl. auch BiRnBACHER (1995, 123 ff.), SCHÖNE-SEIFERT (1997, 219) sowie GutMANN (2002, 179).

19 Dies zeigt Kagan (1988).

20 Ein wesentlicher Unterschied zwischen den in diesem Absatz geschilderten Fällen und solchen der Sterbehilfe besteht natürlich darin, dass das Herbeiführen des Todes im Falle der Sterbehilfe mit dem Willen des Patienten übereinstimmt. Dies deutet darauf hin, dass eine moralische Differenzierung zwischen Fall 1 (Unterlassung von Wiederbelebungsmaßnahmen) und Fall 2 (Verabreichen einer tödlichen Injektion) in den unterschiedlichen Auswirkungen des übereinstimmenden Patientenwillens in diesen beiden Fällen begründet liegen könnte. Wie ich im Folgenden darlegen werde, halte ich diese Überlegung in der Tat für überzeugend; sie ist jedoch davon unabhängig, dass in Fall 1 der Tod des Patienten die Folge eines nicht aufgehaltenen, unabhängigen Prozesses ist. 
scheidung zwischen aktiver und passiver Sterbehilfe aus moralischer Sicht nicht relevant ist? ${ }^{21}$ Dieser Schluss wäre voreilig, denn er setzt voraus, dass der Status des Prozesses, auf den der Tod des Patienten zurückgeführt werden kann, der einzige Aspekt ist, der an der so verstandenen aktiv/passiv-Unterscheidung von Belang ist. Dass dem nicht so ist, wird deutlich, wenn man der Frage nachgeht, warum Fälle passiver Sterbehilfe überhaupt gerechtfertigt sind. Dies führt uns zu dem zweiten der oben genannten Aspekte.

\section{Nicht aufhalten vs. nicht eingreifen}

Bei den Maßnahmen, die für die Erhaltung oder Verlängerung des Lebens des Patienten notwendig sind und auf die im Falle einer passiven Sterbehilfe verzichtet wird, handelt es sich in aller Regel um Eingriffe in den Körper oder die Freiheit des Patienten, die seiner Einwilligung bedürfen, um zulässig zu sein. Die zentrale Relevanz der informierten Einwilligung als wesentliche Ausprägung des Selbstbestimmungsrechtes des Patienten ist in der Medizinethik und auch in der Rechtswissenschaft unbestritten. ${ }^{22}$ Demnach ist ein eigenmächtiges Behandeln ohne (oder gar gegen) den Willen des Patienten bis auf wenige Ausnahmen unzulässig. ${ }^{23}$ Dieses Einwilligungserfordernis gilt unabhängig davon, ob der in Frage stehende Eingriff medizinisch indiziert oder gar überlebensnotwendig ist.

Voraussetzung für eine wirksame Einwilligung ist stets, dass diese bestimmten Gültigkeitskriterien genügt. Dazu gehört, dass der Patient über alle wesentlichen Merkmale des Eingriffs, zu denen insbesondere auch die mit dem Eingriff sowie mit seiner Unterlassung verbundenen Chancen und Risiken gehören, informiert ist, dass die Einwilligung freiwillig und ohne Zwang erfolgt sowie dass der Patient zum Zeitpunkt der Entscheidung für oder gegen eine Einwilligung kompetent ist, eine solche Entscheidung zu treffen. Zudem kann eine einmal erteilte Einwilligung jederzeit

$21 \mathrm{Zu}$ diesem Ergebnis kommen u. a. BIRNBACHER (1995, 348 ff.) und SCHÖNE-SEIFERT (1997, 217 ff.).

22 Vgl. nur BeAuchamp/Childress (2013, 120 ff.), SCHÖNE-SEIFERT (2007, 39 ff.), SCHRoth (2010, 23 ff.) sowie BundESÄrZTEKAMMER (2011b, \S 7 I, 8).

23 Die Ausnahmen betreffen Notfallsituationen, in denen der Wille des Patienten beispielsweise wegen Bewusstlosigkeit aktuell nicht festzustellen, aber eine Behandlung notwendig ist, um großen Schaden vom Patienten abzuhalten. Aber auch in solchen Fällen ist die Behandlung nur dann und insofern gerechtfertigt, als sie dem mutmaßlichen Willen des Patienten entspricht und also nicht gegen seinen Willen erfolgt. Wenn ohne jeden Zweifel bekannt ist, dass der Patient Maßnahmen wie Bluttransfusionen etwa aus religiösen Gründen grundsätzlich ablehnt, dann wäre dies auch im Rahmen einer Notfallbehandlung des bewusstlosen Patienten unzulässig. 
wieder zurückgezogen werden.24 Anlass zu Zweifeln an der Kompetenz zur Einwilligung können beispielsweise kognitive Beeinträchtigungen oder akuter emotionaler Stress geben. Der bloße Umstand, dass ein Patient die Einwilligung in einen lebenserhaltenden bzw. -verlängernden Eingriff verweigert (oder zurückzieht) und sich so für eine Verkürzung seines Lebens entscheidet, ist allerdings kein hinreichendes Kriterium, um diesem Patienten die erforderliche Kompetenz abzusprechen. Folglich ist auch eine medizinische Maßnahme, die der Erhaltung oder Verlängerung des Lebens eines Patienten dient, in der Regel unzulässig, wenn keine gültige Einwilligung des Patienten vorliegt.

Der Verzicht auf oder Abbruch einer lebenserhaltenden Maßnahme im Rahmen einer passiven Sterbehilfe ist also schon allein deshalb gerechtfertigt, weil eine solche Maßnahme unzulässig ist, wenn der Patient seine Einwilligung verweigert bzw. zurückzieht. ${ }^{25}$ Es kommt bei der Rechtfertigung passiver Sterbehilfe somit gar nicht darauf an, dass ein unabhängiger oder in irgendeiner Hinsicht natürlicher, zum Tod führender Prozess nicht länger aufgehalten wird. Dies erklärt lediglich, warum der Patient infolge des Verzichts bzw. Abbruchs stirbt und es sich darum um einen Fall von Sterbehilfe handelt. Für die Rechtfertigung des Verzichts auf die Maßnahme (bzw. ihres Abbruchs) ist nicht erheblich, was durch sie aufgehalten würde, oder dass überhaupt etwas aufgehalten würde, sondern wie sie es aufhalten würde, nämlich durch einen Eingriff, der ohne die wirksame Einwilligung des Patienten unzulässig ist.

Hierin unterscheiden sich passive und aktive Sterbehilfe. Passive Sterbehilfe ist legitim, weil die zu unterlassende oder abzubrechende Maßnahme einwilligungsbedürftig ist, aber nicht bzw. nicht länger dem Willen des Patienten entspricht. Während bei der passiven Sterbehilfe also dem Willen des Patienten entsprechend darauf verzichtet wird, in dessen körperliche Unversehrtheit einzugreifen, besteht die aktive Sterbehilfe in genau so einem Eingriff. Auf diesen Unterschied haben unter anderem Norbert Hoerster sowie Bernard Gert und Kollegen hingewiesen. ${ }^{26}$ Befürworter des Äquivalenzargumentes lassen ihn jedoch zumeist außer Acht. Sie können darauf verweisen, dass der Eingriff bei der aktiven Sterbehilfe wiederum dem Willen des Patienten entspricht, der diese ja freiwillig erbittet. Wie Befürworter der aktiven Sterbehilfe zu Recht betonen, spielt somit auch in der Rechtfertigung aktiver Sterbehilfe eine zentrale Rolle, dass diese mit dem Patientenwillen übereinstimmt. Es liegt deshalb die Frage nahe, wie tief der hier identifizierte Unterschied zwischen aktiver und passiver Sterbehilfe tatsächlich geht. Geht es nicht in beiden Fällen

${ }^{24}$ Auch ein solches Zurückziehen muss jedoch bestimmten Kriterien genügen, um wirksam zu sein.

25 Die zentrale Rolle, die die Einwilligungsbedürftigkeit aller medizinischen Maßnahmen bei der Legitimation von Sterbehilfe durch Behandlungsverzicht bzw. -abbruch spielt, ist weithin anerkannt, vgl. NATIONALER ETHIKRAt (2006, 41), BundesgerichtshoF (2010, Rn. 35), Roxin (2010, 92 f.) und Sumner (2011, Kap. 2).

26 Siehe Hoerster (1986, 65 f.) und GerT ET AL. (2006, 315 f.) 
darum, das Selbstbestimmungsrecht des Patienten zu achten?27 Um diese Frage beantworten $\mathrm{zu}$ können, ist es hilfreich, die moralische Relevanz des Patientenwillens und die spezielle Rolle, die Einwilligungen als Ausdruck dieses Willens spielen, etwas allgemeiner in den Blick zu nehmen. Dies wird es mir erlauben zu zeigen, dass hier tatsächlich ein moralisch relevanter Unterschied zwischen passiver und aktiver Sterbehilfe besteht.

\section{Die Rolle von Einwilligungen}

In der Tat kommt auch aktive Sterbehilfe überhaupt nur in Betracht, wenn sie dem Willen des Patienten entspricht. Die Rolle der Einwilligung ist hier allerdings eine ganz andere: Sie wird gegeben, nicht verweigert. Während sich aus der Verweigerung der Einwilligung in einen Eingriff allerdings dessen Unzulässigkeit und somit ein Anspruch auf sein Unterlassen ergibt, leitet sich aus der Einwilligung in eine Handlung im Allgemeinen noch kein Anspruch auf die Handlung ab. Eine Einwilligung hat - sofern sie den Gültigkeitskriterien genügt (s. oben) - die Erlaubtheit der Handlung zur Folge, sorgt aber im Allgemeinen nicht dafür, dass sie moralisch gefordert ist. ${ }^{28}$ Diese asymmetrische Relevanz von Einwilligungen zeigt sich auch in nicht-medizinischen Bereichen, in denen die Einwilligung eine zentrale Rolle für die moralische Bewertung von Handlungen spielt. Jemanden ins Gesicht zu schlagen ist ohne dessen Einwilligung moralisch wie rechtlich unzulässig; wer es dennoch tut, verletzt einen legitimen Anspruch des Geschlagenen. Willigt eine Person jedoch etwa im Rahmen eines Boxkampfes in Schläge ein, sind diese zulässig (wiederum: sofern die Einwilligung den Gültigkeitskriterien genügt). Gefordert sind sie allerdings nicht, zumindest nicht aufgrund der Einwilligung. Einem Fremden auf der Straße meine Einwilligung zu geben, mich $\mathrm{zu}$ schlagen, berechtigt mich schließlich nicht, anschließend die Schläge von ihm einzufordern. Gleiches gilt für die Einwilligung in sexuelle Handlungen: Ohne Einwilligung sind diese unzulässig und es besteht ein Anspruch auf Unterlassung, aber natürlich gibt weder die eigene Einwilligung noch die des anderen jemandem ein Recht, sexuelle Handlungen einzufordern.

Dies hängt damit zusammen, wie die Einwilligung des von einer Handlung Betroffenen die normative Situation des Handelnden ändert. In Fällen wie dem eines Schlages ins Gesicht spricht etwas Gewichtiges gegen die Handlung, wenn der Be-

27 Diese Überlegung findet sich etwa bei SUMNER $(2011,90)$.

28 In einzelnen Fällen ist es allerdings durchaus möglich, dass eine Handlung, die ohne (tatsächliche oder mutmaßliche) Einwilligung unzulässig wäre, bei vorliegender Einwilligung gefordert ist, etwa das Verabreichen einer bereitstehenden, lebensrettenden Blutkonserve. Dies liegt jedoch nicht in der Einwilligung selbst begründet, sondern in den gewichtigen Gesichtspunkten, die in einem solchen Fall für die Handlung sprechen. 
troffene nicht eingewilligt hat: Sie verursacht Schmerzen und greift in die körperliche Unversehrtheit ein. Gleiches gilt für medizinische Maßnahmen. Auch diese gehen in der Regel mit Schmerzen oder Eingriffen in die körperliche Integrität einher, weshalb sie in der deutschen Rechtsprechung üblicherweise als Körperverletzung gewertet werden. ${ }^{29}$ Die Einwilligung des Betroffenen sorgt nun allerdings nicht dadurch für die Zulässigkeit solcher Handlungen, dass sie diesen Gründen gegen die Handlung solche entgegensetzt, die für die Handlung sprechen. Stattdessen hat sie zur Folge, dass einige oder alle der zuvor problematischen Gesichtspunkte außer Kraft gesetzt werden und somit nicht länger gegen die Handlung sprechen. Metaphorisch gesprochen: Die Einwilligung fügt nicht der „Für“-Waagschale etwas hinzu, das dann den Inhalt der „Gegen“-Waagschale überwiegt, sondern nimmt etwas aus der „Gegen“-Waagschale heraus, so dass diese nicht mehr schwerer wiegt. Zwei Überlegungen machen dies deutlich.

Erstens ist es bei anderen leidensmindernden medizinischen Eingriffen wie beispielsweise dem Einsetzen eines künstlichen Kniegelenkes irreführend, diese so zu beschreiben, dass ohne die Einwilligung des Patienten noch nicht genug für den Eingriff spricht, mit der Einwilligung dagegen schon. Und auch wenn ein Patient aus religiösen Gründen seine Einwilligung in eine lebensrettende Operation verweigert, weil damit eine Bluttransfusion verbunden wäre, wäre es unangemessen, die Situation als eine zu beschreiben, in der noch nicht genug für den Eingriff spricht, um dessen Durchführung insgesamt zur moralisch besseren Option zu machen. Stattdessen spricht ohne die Einwilligung etwas sehr Gewichtiges gegen diese Eingriffe, das durch die Einwilligung allerdings außer Kraft gesetzt wird. Nach Erteilen der Einwilligung spricht also nicht unbedingt mehr für, sondern vor allem weniger gegen die entsprechende Handlung.

Zweitens ist der bloße Verweis auf die Einwilligung des Betroffenen einer Handlung nicht geeignet, diese Handlung auch zu rechtfertigen. Angenommen, A willigt ein, dass B ihn ins Gesicht schlägt. Wenn B dies nun tut und anschließend von $\mathrm{C}$ gefragt wird, warum er A geschlagen habe, ist „,weil er eingewilligt hat" keine geeignete Antwort, und zwar nicht bloß, weil sie auf einen Grund verweist, der nicht hinreichend ist, um die Handlung zu rechtfertigen (wie es der Fall wäre, wenn A nicht eingewilligt hätte und B mit „weil ich Lust hatte“ antworten würde). Die Antwort benennt überhaupt keinen Grund und ist deswegen grundsätzlich ungeeignet, eine solche Handlung zu rechtfertigen. ${ }^{30}$ Würde die Einwilligung selbst einen Grund für den Schlag liefern, so würde B, wenn er trotz der Einwilligung nicht zu-

29 Vgl. FISCHER (2014, \$ 223 Rn. 17).

30 Sie mag abhängig vom Kontext freilich implizit auf einen nicht genannten Grund hinweisen. Ist es zwischen B und C geteiltes Wissen, dass B den A nicht leiden kann, und beantwortet B die Frage von C in der genannten Weise, begleitet von einem Grinsen, so mag dies darauf verweisen, dass B den A schon immer mal ins Gesicht schlagen wollte. Auch dann rechtfertigt aber nicht die explizit benannte Einwilligung des A, sondern (wenn überhaupt) der implizit kommunizierte Wunsch B's die Handlung. 
schlägt, nun einem Grund zuwiderhandeln. Es spricht aber augenscheinlich trotz Einwilligung überhaupt nichts dagegen, auf den Schlag zu verzichten, solange nicht ein anderer Gesichtspunkt benannt werden kann, der für den Schlag spricht. Die Einwilligung des A stellt also selbst keinen positiven Grund bereit, der B's Schlag rechtfertigen könnte.

Man kann dies so zusammenfassen, dass die Einwilligung in eine Handlung keinen positiven, sondern lediglich einen negativen Beitrag zu deren Rechtfertigung leistet, indem sie einige oder alle gegen die Handlung sprechenden Gesichtspunkte, die es durch eine positive Rechtfertigung aufzuwiegen gilt, außer Kraft setzt. Das erklärt, warum sich allein mit einer Einwilligung in eine einwilligungsbedürftige Handlung noch keine Forderung begründen lässt, denn eine solche Forderung setzt voraus, dass eine positive Rechtfertigung für die Handlung verfügbar ist, was die Einwilligung selbst jedoch nicht garantiert. Das Zurückziehen einer solchen Einwilligung hingegen setzt die problematischen Gesichtspunkte der Handlung, auf die sich eine Unterlassungsforderung berufen kann, wieder in Kraft.

Diese asymmetrische Relevanz von Einwilligungen wird verschleiert, wenn davon die Rede ist, dass eine medizinische Maßnahme oder deren Abbruch „dem Willen des Patienten entspricht“" oder dass bei der Durchführung solcher Maßnahmen die „Entscheidungen des Patienten“ zu respektieren sind, denn dadurch wird der Unterschied zwischen dem Geben und dem Verweigern einer Einwilligung ausgeblendet. ${ }^{31}$ Solche Formulierungen laden außerdem zu einer Verwechslung von zwei Aspekten der Selbstbestimmung einer Person ein, die es zu unterscheiden gilt. Damit, dass es A's Willen oder seiner Entscheidung entspricht, von B geschlagen zu werden, kann zweierlei gemeint sein, nämlich zum einen A's Einwilligung in den Schlag und zum anderen sein Wunsch, von B geschlagen zu werden (den er zudem als Bitte äußern mag). Letzterer mag B einen Grund für den Schlag liefern, denn anders als Einwilligungen können Wünsche durchaus einen positiven Beitrag zur Rechtfertigung von Hilfe bei ihrer Erfüllung leisten. Wünschen fehlt allerdings der negative Rechtfertigungsbeitrag von Einwilligungen: Sie setzen Gesichtspunkte, die gegen ihre Erfüllung sprechen, nicht einfach außer Kraft. Wichtig ist zudem, dass die Einwilligung in eine Handlung zwar häufig - und im Kontext medizinischer Behandlungen wohl fast immer -, aber nicht notwendigerweise mit dem Wunsch, dass diese Handlung ausgeführt wird, einhergeht. Dies zeigt das Beispiel des Boxers, der in die Schläge seines Gegners eingewilligt hat, aber dennoch nicht von ihm geschlagen zu werden wünscht und seinen Schlägen darum zu entgehen versucht. ${ }^{32}$

Es ist deshalb zwar angesichts der weitgehenden Übereinstimmung zwischen Wunsch und Einwilligung im Bereich der Medizin durchaus nachvollziehbar, dass gerade im Kontext der Medizinethik die moralische Relevanz von Einwilligungen

31 Vgl. Gert ET AL. (1994).

32 Vgl. dazu ausführlicher MüLLER (i.E., \3). Auf den Unterschied zwischen der Einwilligung in eine Handlung und dem Wunsch nach oder der Bitte um die Ausführung der Handlung weisen auch Owens $(2012,173)$ bzw. Dempsey $(2013,17)$ hin. 
mit der Relevanz der Wünsche und Bitten des Patienten gleichgesetzt wird. Dadurch wird aber genau jene Asymmetrie unterschlagen, die erklärt, warum es zwar keinen prinzipiellen moralischen Unterschied zwischen der Nichterfüllung eines Wunsches nach Unterlassung einer Maßnahme und der eines Wunsches nach Durchführung einer Maßnahme gibt, es aber dennoch sehr viel problematischer ist, eine Maßnahme ohne Einwilligung des Patienten durchzuführen, als trotz Einwilligung des Patienten von einer Maßnahme abzusehen. Ist dieses Missverständnis einmal offengelegt, ist auch ersichtlich, dass es einen bedeutenden moralischen Unterschied macht, ob die Willensäußerung des Patienten im Erteilen oder Verweigern einer Einwilligung besteht, auch wenn sie in beiden Fällen mit einem entsprechenden Wunsch des Patienten einhergeht. Dieser Unterschied trennt aktive und passive Sterbehilfe, obwohl man sich in der Rechtfertigung beider auf den Willen und das Selbstbestimmungsrecht des Patienten berufen kann.

\section{Zwischenfazit}

Die vorangegangenen Überlegungen machen deutlich, dass es sehr wohl einen moralisch relevanten Unterschied zwischen aktiver und passiver Sterbehilfe gibt, denn nur bei der passiven Sterbehilfe wird auf die (weitere) Durchführung einer einwilligungsbedürftigen Maßnahme verzichtet, weil der Patient seine Einwilligung verweigert bzw. zurückzieht. Hieraus ergibt sich ein legitimer Anspruch des Patienten auf den Abbruch der Maßnahme und damit auf die Durchführung der Sterbehilfe, die in solchen Fällen somit nicht bloß zulässig, sondern geboten ist der Patient hat ein Recht darauf, dass die Maßnahme nicht durchgeführt bzw. abgebrochen wird, wenn er seine Einwilligung verweigert oder zurückzieht. Die Zulässigkeit passiver Sterbehilfe lässt sich folglich unter Rückgriff auf eine Rechtfertigungsressource begründen, die für Fälle aktiver Sterbehilfe nicht zur Verfügung steht, denn das Unterlassen der zum Tod führenden Handlung im Falle der aktiven Sterbehilfe (z.B. das Vorenthalten der tödlichen Spritze) ist nichts, das der Einwilligung des Patienten bedarf. Damit hat sich gezeigt, dass die Äquivalenzthese bezüglich aktiver und passiver Sterbehilfe zurückgewiesen werden muss; zwischen ihnen besteht ein moralisch relevanter Unterschied. Folglich scheitert auch das Äquivalenzargument für die Zulässigkeit aktiver Sterbehilfe - daraus, dass passive Sterbehilfe zulässig ist, folgt nicht, dass auch aktive Sterbehilfe in manchen Situationen zulässig sein muss.

Was heißt das für die Zulässigkeit der aktiven Sterbehilfe? Es scheint, als sei mit der Zurückweisung der Äquivalenzthese zugleich der erste Schritt zur Begründung der restriktiven Position getan. Tatsächlich hat sich damit zwar eine der Voraussetzungen der restriktiven Position - dass es einen moralisch relevanten Unterschied zwischen aktiver und passiver Sterbehilfe gibt - als richtig herausgestellt. Mit Blick auf das Ziel, die Unzulässigkeit aktiver Sterbehilfe zu begründen, sind Vertreter der 
restriktiven Position allerdings keinen Schritt weiter, denn der Unterschied besteht nicht darin, dass im Falle der aktiven Sterbehilfe ein (weiterer) gegen sie sprechender Gesichtspunkt vorliegt, von dem sich fragen ließe, ob er moralisch entscheidend ist und den Ausschlag gegen die Zulässigkeit aktiver Sterbehilfe gibt. Der Umstand, dass es sich bei der aktiven Sterbehilfe nicht um das Respektieren der Verweigerung der Einwilligung in einen Eingriff handelt, spricht ja nicht gegen sie. Mehr noch: Die Ergebnisse des letzten Abschnittes erlauben es, die Herausforderung, der sich Verteidiger der restriktiven Position ausgesetzt sehen, zu verschärfen und zugleich zu zeigen, wie ein direktes Argument, das die Zulässigkeit der aktiven Sterbehilfe unter Verweis auf das Selbstbestimmungsrecht zu begründen beansprucht, präzisiert und gegen zwei verbreitete Einwände verteidigt werden kann.

\section{Selbstbestimmung und die Zulässigkeit aktiver Sterbehilfe}

Wir haben gesehen, dass nur die Verweigerung der Einwilligung in eine medizinische Maßnahme den Patienten automatisch mit einem Anspruch auf den Verzicht auf die Maßnahme ausstattet und deshalb ein Unterschied in den zur Verfügung stehenden Rechtfertigungsressourcen zwischen aktiver und passiver Sterbehilfe besteht. Die Kehrseite dieser Feststellung ist, dass Einwilligungen grundsätzlich Handlungen legitimieren können, die andernfalls moralisch unzulässig wären. Sonst wäre nicht ersichtlich, warum künstliche Beatmung, Wiederbelebung und andere Maßnahmen mit Einwilligung des Patienten zulässig sind. Wie in Abschnitt 5 erläutert, bewirken Einwilligungen eine solche Veränderung der normativen Situation des Handelnden, indem sie gegen die Handlung sprechende Gesichtspunkte außer Kraft setzen. Dies alles muss auch der Verfechter einer restriktiven Position akzeptieren, der zur Rechtfertigung seiner unterschiedlichen Beurteilung von aktiver und passiver Sterbehilfe die asymmetrische Relevanz von Einwilligungen heranzuziehen versucht.

Auf die Möglichkeit, eine ansonsten unzulässige Handlung zu erlauben, indem man als Betroffener dieser Handlung in sie einwilligt, verweisen jedoch auch die Vertreter eines direkten Argumentes für die Zulässigkeit der aktiven Sterbehilfe. Sie berufen sich oft auf das Selbstbestimmungsrecht des Patienten, das es auch bei Entscheidungen über Leben und Tod zu respektieren gelte. Die Rede von Selbstbestimmung ist jedoch, wie wir im fünften Abschnitt gesehen haben, mehrdeutig. Es ist deshalb sinnvoll, nicht von einem Selbstbestimmungsargument, sondern von einem Einwilligungsargument für die Zulässigkeit aktiver Sterbehilfe zu sprechen. Die Einwilligung sorgt im oben diskutierten Falle des Boxers dafür, dass die entsprechende Handlung moralisch zulässig ist, und auch sexuelle Handlungen sind im Allgemeinen zulässig, wenn eine gültige Einwilligung aller Beteiligten vorliegt. Warum nun, so die dem Einwilligungsargument zugrundeliegende Überlegung, 
sollte die Einwilligung des Patienten in Fall 2 nicht ebenfalls ausreichen, um die tödliche Injektion zwar nicht moralisch gefordert, aber doch zumindest zulässig zu machen?

Verfechter einer restriktiven Position können dieser Konsequenz nur entgehen, indem sie die Reichweite dieses Einwilligungsvermögens - und damit die Selbstbestimmung des Patienten - beschränken. Genauer gesagt: Sie müssen annehmen, dass die Einwilligung des Patienten manche, aber nicht alle Gesichtspunkte, die gegen eine Maßnahme sprechen können, außer Kraft zu setzen vermag. Im Gegensatz beispielsweise zu den Verletzungen des Boxers durch die Schläge seines Gegners liegen einige solcher Gesichtspunkte demzufolge jenseits der Reichweite wirksamer Einwilligungen. Zudem müssen sie zeigen, dass ein solcher Gesichtspunkt in allen Fällen aktiver Sterbehilfe die Gründe, die für die Sterbehilfe sprechen, überwiegt. Aktive Sterbehilfe ist also trotz Einwilligung des Patienten nur dann grundsätzlich unzulässig, wenn in allen Fällen, in denen diese zur Entscheidung steht, ein Gesichtspunkt vorliegt, für den gilt, dass er zum einen einwilligungsresistent ist und zum anderen alle Gründe, die in der jeweiligen Situationen für die Sterbehilfe sprechen, zu überwiegen vermag.

Welcher Gesichtspunkt kann diese anspruchsvolle Rolle spielen? Ist es der Umstand, dass aktive Sterbehilfe ärztliches Handeln beinhaltet, welches den Tod des Patienten zeitlich unmittelbar kausal zur Folge hat? Dies gilt auch für das Ausschalten einer Beatmungsmaschine. Oder ist es der Umstand, dass der Patient nicht infolge eines natürlichen Prozesses stirbt? Die moralische Relevanz dieses Gesichtspunktes hat sich bereits in Abschnitt 3 als zweifelhaft herausgestellt. Verfechter der restriktiven Position mögen an dieser Stelle einfach darauf pochen, dass menschlichem Leben ein unbedingter und nicht abzuwiegender Wert zukommt, der es selbst für den, dessen Leben es ist, gänzlich unverfügbar macht. Eine solche These bedarf jedoch einer plausiblen Begründung. Dass eine solche Begründung angesichts der Bedingung, dass sie, um allgemein überzeugen zu können, auf weltanschaulich-religiöse Annahmen verzichten müsste, nicht in Sicht ist, ist an anderer Stelle überzeugend dargelegt worden. ${ }^{33}$

Zudem würde dieser Gesichtspunkt auch gegen passive Sterbehilfe wie in Fall 3 sprechen. Auch wer ein lebenserhaltendes Beatmungsgerät abstellt, verfügt über das Leben eines anderen, wenn auch auf dessen Geltendmachen eines berechtigten Anspruchs hin. ${ }^{34}$ Wer dies für zulässig hält, gesteht also zu, dass ein solcher Wert

33 Vgl. Kuhse (1987), Dworkin (1993, Kap. 3) und QuAnte (2002, 239 ff.).

34 Gleiches gilt für den Versuch, die grundsätzliche Unzulässigkeit der aktiven Sterbehilfe mithilfe eines Rechts auf Leben des Patienten zu begründen, über das dieser (anders als etwa über sein Recht auf körperliche Unversehrtheit) nicht verfügen kann. Auch dieses würde in Fall 3 verletzt. Zudem ist schwer zu sehen, wie ein solches Recht gewissermaBen gegen den Willen des Rechteinhabers gewendet werden kann, um dessen Verfügungsfreiheit einzuschränken und ihm im Ergebnis eine Pflicht zum Leben aufzuerlegen. Dies scheint mit einem Verständnis dieses Rechts als eines Abwehrrechts inkom- 
zumindest den Anspruch auf das Abbrechen einer lebenserhaltenden Maßnahme, der sich aus der Einwilligungsbedürftigkeit dieser Maßnahme ergibt, nicht zu überwiegen vermag, sondern vielmehr selbst durch diesen überwogen wird. Daraus folgt zwar nicht schon, dass jener Wert auch in Fällen aktiver Sterbehilfe überwogen werden kann, denn ein solcher Anspruch kann bei aktiver Sterbehilfe ja gerade nicht zur Rechtfertigung herangezogen werden. Warum kann dieser Wert dann aber nicht auch etwa durch das als unerträglich empfundene und nur durch Herbeiführung des Todes vermeidbare Leid eines Patienten überwogen werden?

Hier drängt sich der Verdacht auf, dass der Vertreter der restriktiven Position sich die spezifische Relevanz dieses Wertes ad hoc so zurechtlegt, dass er genau die Rolle spielt, die im Rahmen seiner Position erforderlich ist. Eine überzeugende Begründung dieser Position müsste stattdessen erklären, warum dieser Wert alle anderen Gesichtspunkte bis auf genau einen überwiegen können und warum er anders als so viele andere Gesichtspunkte grundsätzlich einwilligungsresistent sein sollte. Der Vertreter der restriktiven Position ist hier in der Bringschuld: Kann er nicht plausibel machen, dass gegen die aktive Sterbehilfe derart schwergewichtige Gesichtspunkte sprechen, die grundsätzlich außerhalb der Reichweite wirksamer Einwilligungen liegen, gibt es vor dem Hintergrund des in Abschnitt 5 dargelegten Verständnisses der Rolle von Einwilligungen keinen überzeugenden Grund, die Gültigkeit des Einwilligungsargumentes für die Zulässigkeit zumindest mancher Fälle aktiver Sterbehilfe zu bezweifeln.

\section{Zwei Einwände}

Gegen derartige Argumente, die sich auf das Selbstbestimmungsrecht des Patienten berufen, sind jedoch andere Einwände vorgebracht worden, die auch viele derer skeptisch machen, die aktive Sterbehilfe in manchen Situationen für zulässig halten. Denn, so der erste Einwand, wenn allein die Einwilligung des Patienten hinreicht, um eine aktive Sterbehilfe zu rechtfertigen, dann muss dies auch in den Fällen gelten, in denen ein Patient nicht aufgrund von Schmerzen oder anderem Leid, sondern beispielsweise aus bloßer „Lebenssattheit“ sterben will. Dies aber geht vielen zu weit.

Dieser Einwand verliert jedoch an Schlagkraft, wenn man sich in Erinnerung ruft, dass eine Einwilligung keine positive Rechtfertigung für eine Handlung bereitstellt, sondern lediglich einen negativen Beitrag zu ihrer Rechtfertigung leistet, in-

patibel zu sein und stattdessen vorauszusetzen, dass es nicht die Freiheit des Inhabers, sondern einen objektiven Wert von der oben diskutierten Art schützt. 
dem sie gegen die Handlung sprechende Gesichtspunkte außer Kraft setzt. ${ }^{35}$ Gerade so schwerwiegende Eingriffe wie etwa eine tödliche Injektion aber erfordern eine positive Rechtfertigung durch gute Gründe. Nun ist auch in problematischen Fällen wie bloßer Lebenssattheit anzunehmen, dass der Patient nicht nur in die Sterbehilfe einwilligt, sondern diese auch wünscht und darum bittet; und anders als Einwilligungen können solche Wünsche und Bitten grundsätzlich zur positiven Rechtfertigung einer Handlung beitragen (vgl. Abschnitt 5).

Sie tun dies jedoch nur, wenn sie bestimmten Bedingungen genügen, die nicht in jedem Fall bei einer grundsätzlich einwilligungsfähigen Person erfüllt sind. Insbesondere sind die Bedingungen dafür, dass die Wünsche eines anderen mir einen Grund geben, aktiv zu ihrer Erfüllung beizutragen, anspruchsvoller als die Bedingungen, die erfüllt sein müssen, damit ich seine Wünsche respektieren muss und ihn nicht bei ihrer Erfüllung behindern darf. Aus Erwägungen des Wohlwollens etwa ergibt sich im ersten Fall nur dann ein Grund zu helfen, wenn anzunehmen ist, dass die Wünsche mit dem Wohlergehen der Person übereinstimmen, was nicht immer der Fall ist. Diese Bedingung muss meines Erachtens jedoch nicht erfüllt sein, um Wünsche im genannten Sinne respektabel zu machen. Angenommen ein Freund wünscht, eine großflächige Tätowierung seiner Unterarme vornehmen zu lassen, weil er glaubt, dass dies ihn insgesamt glücklicher machen wird, liegt damit jedoch in vorhersehbarer Weise falsch. Dann kann es geboten sein, seinen Wunsch dennoch zu respektieren und nicht in paternalistischer Weise zu versuchen, die Tätowierung zu verhindern. Der Wunsch gibt mir jedoch keinen Grund, bei seiner Erfüllung etwa durch die Finanzierung der Tätowierung behilflich zu sein. Ebenso wenig gibt der Wunsch zu sterben jemandem einen Grund, bei der Herbeiführung des Todes behilflich zu sein, wenn er offensichtlich einer Fehleinschätzung des zu erwartenden künftigen Wohlergehens entspringt.

Doch selbst wenn der Sterbewunsch derartige Bedingungen nicht verletzt, heißt das noch nicht, dass damit das entsprechende Handeln eines Arztes bereits als unproblematisch ausgewiesen ist. Dies kann zumindest zurückweisen, wer die Gründe, aus denen ein Arzt handeln darf, gegenüber denen anderer Personen für beschränkt hält. In der Tat halten wir in vielen Kontexten das Handeln aus manchen Gründen, die für andere eine Handlung zu rechtfertigen vermögen, im Falle von Ärzten für unzulässig oder zumindest problematisch. Beispiele hierfür sind Profitmaximierung oder die Befriedigung sexueller Bedürfnisse. Aus solchen Gründen, so scheint es, sollten Ärzte nicht (be-)handeln. Viele lehnen zudem ein Bild von Ärzten als bloßen Wunscherfüllern ab, die den Bitten eines Patienten um die Durchführung bestimmter Maßnahmen unabhängig davon nachkommen, ob sie diese für medizinisch sinnvoll halten. Vor dem Hintergrund eines so verstandenen ,ärztlichen Handlungs-

35 Natürlich leistet eine Einwilligung dies auch nur dann, wenn sie den Gültigkeitskriterien genügt. Es ist meines Erachtens zu bezweifeln, dass dies in vielen der Fälle zutreffen wird, in denen ein Patient ohne (aktuelle oder antizipierte) Schmerzen oder andere Leiden um aktive Sterbehilfe bittet. 
ethos“ ließe sich also eine Begrenzung der Zulässigkeit aktiver Sterbehilfe begründen, die Fälle bloßer Lebenssattheit grundsätzlich ausschließt.

Der Sorge, eine auf dem Einwilligungsargument aufbauende Rechtfertigung aktiver Sterbehilfe laufe unweigerlich darauf hinaus, dass diese zulässig sei, wann immer ein Patient sie wünscht und seine Einwilligung erteilt, kann somit begegnet werden. Die Zulässigkeit aktiver Sterbehilfe in Fällen, in denen Leid und Schmerzen, die aus Sicht des Patienten unerträglich sind, Anlass für seinen Wunsch zu sterben sind, wird durch solche Überlegungen jedoch nicht in Frage gestellt. Schließlich gehört das Beenden oder Verhindern von Schmerzen und Leid seit jeher zu den Kernaufgaben eines Arztes. In solchen Fällen liegen also durchaus paradigmatische Gründe für ärztliches Handeln vor.

An diese Überlegungen lässt sich jedoch ein zweiter Einwand anschließen: Wenn das Beenden von Leid und Schmerz auch dann für ein ärztliches Handeln spricht, wenn dieses im Herbeiführen des Todes eines Patienten besteht, ist es dann nicht auch prinzipiell möglich, dass es die gegen solche Tötungen sprechenden Gesichtspunkte überwiegt, wenn diese nicht durch die Einwilligung des Patienten außer Kraft gesetzt werden? Läuft dies also darauf hinaus, dass auch unfreiwillige „Mitleidstötungen“ gerechtfertigt sein können?

Dieser Befürchtung lässt sich zum einen entgegensetzen, dass nichts des bisher Gesagten das Prinzip in Frage stellt, demzufolge es grundsätzlich unzulässig ist, eine Person gegen ibren Willen zu töten, selbst dann, wenn sie dadurch von einem Leid „erlöst“" würde. Zum anderen ignoriert die Befürchtung die Rolle, die der Wille des Patienten bei der Bestimmung seines Leidens spielt. Dass ein Patient einem Zustand zu entgehen wünscht, ist zwar nicht hinreichend, um davon zu sprechen, dass er unter diesem Zustand leidet (andernfalls wäre jede Erfüllung eines Wunsches zugleich das Beseitigen eines Leids). Andererseits bestehen hinsichtlich der Überzeugung, dass ein Patient an einem Zustand leidet, erhebliche Rechtfertigungsschwierigkeiten, wenn der Patient in diesem Zustand zu verbleiben wünscht. Dass eine Person etwas Vermeidbares dennoch wünscht, spricht in epistemisch prima facie ausschlaggebender Weise dagegen, dass sie darunter leidet. Wer eine Person gegen ihren Willen aus Mitleid tötet, macht also in der Regel den Fehler, die Person hinsichtlich Ihres vermeintlichen Leidens zu bevormunden, und ist schon deshalb in seinem Handeln nicht gerechtfertigt. ${ }^{36}$ Auch dieser zweite Einwand gegen das Selbstbestimmungs-Argument für die Zulässigkeit aktiver Sterbehilfe geht somit fehl.

36 Die Situation ist komplizierter, wenn das Herbeiführen des Todes eines leidenden Patienten nicht gegen, sondern lediglich obne seinen erklärten Willen erfolgen würde, etwa weil dieser zu einer solchen Willenserklärung nicht (mehr) in der Lage ist und auch keine frühere Erklärung vorliegt. Eine hinreichend differenzierte Auseinandersetzung mit der Frage, ob in solchen Fällen auch eine aktive Sterbehilfe unter bestimmten Bedingungen moralisch zulässig sein kann, würde den Rahmen dieses Textes jedoch überschreiten. 


\section{Schlussbemerkung}

Der hier vorgelegte Text untersucht die Rolle, die verschiedene Aspekte dessen, was man gemeinhin als Selbstbestimmung bezeichnet, bei der Beantwortung der Frage spielen, ob und unter welchen Bedingungen es moralisch zulässig ist, dass Ärzte an der Herbeiführung des Todes von Patienten mitwirken. Diese Untersuchung ist zu einem insbesondere für Vertreter einer restriktiven Position bezüglich der Zulässigkeit aktiver Sterbehilfe zweischneidigen Ergebnis gekommen. Einerseits hat sich gezeigt, dass das gegenwärtig verbreitete Verständnis der Unterscheidung zwischen aktiver und passiver Sterbehilfe, das auf das Kriterium des Behandlungsbezuges abstellt, entgegen mancher Vertreter einer liberalen Position durchaus einen moralisch relevanten Unterschied markiert. Deren Äquivalenzargument für die Zulässigkeit auch aktiver Sterbehilfe scheitert somit.

Andererseits setzt die Begründung, warum hier ein moralisch relevanter Unterschied besteht, ein Verständnis der moralischen Rolle von Einwilligungen voraus, das denjenigen, der im Gegensatz zur passiven die aktive Sterbehilfe für grundsätzlich unzulässig hält, in Bedrängnis bringt. Es zeigt sich nämlich, dass er, wenn er sich diese Begründung zu eigen macht, zugleich den Ausgangspunkt eines direkten Argumentes für die Zulässigkeit aktiver Sterbehilfe akzeptiert. Dessen Konklusion könnte er nur noch entgehen, indem er eine überzeugende und nicht auf weltanschaulich-religiösen Annahmen beruhende Begründung für die These vorlegt, dass in allen Fällen aktiver Sterbehilfe etwas gegen deren Durchführung spricht, das anders als andere solcher Gesichtspunkte grundsätzlich einwilligungsresistent ist und zudem alle Gründe, die für eine solche Maßnahme sprechen können, überwiegt. Eine solche Begründung, die zugleich der Zulässigkeit passiver Sterbehilfe ohne Adhoc-Annahmen Rechnung zu tragen vermag, ist jedoch nicht in Sicht.

Außerdem können mithilfe eines differenzierteren Verständnisses der moralischen Relevanz von Einwilligungen und Wünschen als zwei zu unterscheidenden Aspekten von Selbstbestimmung zwei verbreitete Einwände zurückgewiesen werden: Weder legitimiert eine vom Selbstbestimmungsrecht des Patienten ausgehende Argumentation eine aktive ärztliche Sterbehilfe notwendigerweise auch in Fällen, in denen diese aus bloßer „Lebenssattheit" gewünscht wird, noch zwingt sie uns, in der Konsequenz sogar unfreiwillige „Mitleidstötungen“ für rechtfertigbar zu halten. Insgesamt zeigen diese Überlegungen zur Rolle der Selbstbestimmung also, dass die Zulässigkeit aktiver Sterbehilfe sich zwar nicht schon mithilfe des Äquivalenzargumentes aus der Zulässigkeit passiver Sterbehilfe ergibt, eine liberale Position aber dennoch erheblich besser begründet ist. ${ }^{37}$

37 Für hilfreiche Diskussionen und Kommentare zu früheren Versionen dieses Textes danke ich Johann S. Ach, Susanne Boshammer, Annette Dufner, Jan Gertken, Benjamin Kiesewetter, Felix Koch, Peter Schaber, Thomas Schmidt, Konstantin Schnieder, Bettina Schöne-Seifert, Moritz Schröder, Héctor Wittwer sowie den Teilnehmerinnen und Teilnehmern der Kolloquien von Susanne Boshammer in Osnabrück und Thomas Schmidt an der HU Berlin. 


\section{Literatur}

ACH, J., Wiesing, U., MARCKMANN, G. (2012): Sterbehilfe: Einführung, in: WIESING, U. (Hg.), Ethik in der Medizin, 4. Auflage. Reclam, Stuttgart, 232-243.

Beauchamp, T. L., Childress, J. F. (2013): Principles of Biomedical Ethics, 7. Auflage. Oxford University Press, New York.

BIRNBACHER, D. (1995): Tun und Unterlassen, Reclam, Stuttgart.

Brody, B. A. (1989): Der vegetabile Patient und die Ethik der Medizin, in: SAss, H.-M. (Hg.), Medizin und Ethik, Reclam, Stuttgart, 296-310.

BUNDESÄRZTEKAMMER (2011a): Grundsätze der Bundesärətekammer zur ärұtlichen Sterbebegleitung, Deutsches Ärzteblatt 108(7), A346-348.

- (2011b): (Muster-)Berufsordnung für die in Deutschland tätigen Ärz̧tinnen und Äræte in der Fassung der Beschlüsse des 114. Deutschen Äratetages 2011 in Kiel, [http://www.bundesaerztekammer.de/downloads/MBO_08_20112.pdf].

BUNDESGERICHTSHOF (2010): Urteil vom 25.06.2010 [Sterbehilfe durch Behandlungsabbruch7, BGH 2 StR 454/09.

Dempsey, M. M. (2013): Victimless Conduct and the Volenti Maxim: How Consent Works, Criminal Law and Philosophy 7, 11-27.

Dworkin, R. (1993): Life's Dominion, Random House, New York.

FISCHER, T. (2014): Strafgesetzbuch mit Nebengesetzen, 61. Auflage. Beck, München.

Gert, B., Bernat, J. L., Mogielnicki, R. P. (1994): Distinguishing between Patients' Refusals and Requests, Hastings Center Report 24(4), 13-15.

Gert, B., Culver, C. M., Clouser, K. D. (2006): Bioethics: A Systematic Approach, 2. Auflage. Oxford University Press, New York.

GESANG, B. (2001): Aktive und passive Sterbebilfe - Zur Rehabilitation einer stark kritisierten deskriptiven Unterscheidung, Ethik in der Medizin 13, 161-175.

Gutmann, T. (2002): Der eigene Tod-Die Selbstbestimmung des Patienten und der Schutz. des Lebens in ethischer und rechtlicher Dimension, Ethik in der Medizin 14, 170-185.

HOERSTER, N. (1986): Sterbehilfe im säkularen Staat, Suhrkamp, Frankfurt a. M.

HÖFLING, W., RIXEN, D. (2003): Vormundschaftsgerichtliche Sterbeherrschaft?, Juristenzeitung 58, 884-894.

Kagan, S. (1988): The Additive Fallacy, Ethics 99(1), 5-31.

Kunse, H. (1987): The Sanctity-of-Life Doctrine in Medicine, Clarendon Press, Oxford.

MCInTyre, A. (2014): Doctrine of Double Effect, in: ZaLTa, E. N. (Hg.), Stanford Encyclopedia of Philosophy, Winter 2014 Edition, [http://plato.stanford.edu/archives/win2014/entries/double-effect/]. 
Müller, A. (i.E.): Die Verbindlichkeit von Patientenverfügungen: Welcher Wille zählt?, in: Dietrich, F., MÜller-SAlo, J., SCHMÜCKer, R. (Hg.), Zeit - eine normative Ressource?, Klostermann, Frankfurt a.M.

NATIONALER ETHIKRAT (2006): Selbstbestimmung und Fürsorge am Lebensende. Stellungnabme, Berlin, [http://www.ethikrat.org/dateien/pdf/selbstbestimmung-undfuersorge-am-lebensende.pdf].

OwEns, D. (2012): Shaping the Normative Landscape, Oxford University Press, Oxford.

QuANTE, M. (1998): Passive, indirekt und direkt aktive Sterbehilfe - deskriptiv und ethisch tragfähige Unterscheidungen?, Ethik in der Medizin 10, 206-226.

- (2002): Personales Leben und menschlicher Tod, Suhrkamp, Frankfurt a. M.

RaCHELS, J. (1989): Aktive und Passive Sterbehilfe, in: SASS, H.-M. (Hg.), Medizin und Ethik, Reclam, Stuttgart, 254-264.

ROXIN, C. (2010): Zur strafrechtlichen Beurteilung der Sterbehilfe, in: ROXIN, C., SCHROTH, U. (Hg.), Handbuch des Medizinstrafrechts, 4. Auflage. Boorberg, Stuttgart, 75-121.

SCHÖNE-SEIFERT, B. (1997): Die Grenzen zwischen Töten und Sterbenlassen, in: Honnefelder, L., StrefFer, C. (Hg.), Jahrbuch für Wissenschaft und Ethik, Band 2. De Gruyter, Berlin, 205-226.

- (2007): Grundlagen der Medizinethik, Kröner, Stuttgart.

SCHROTH, U. (2010): Ärztliches Handeln und strafrechtlicher Maßstab, in: Roxin, C., SCHroth, U. (Hg.), Handbuch des Medizinstrafrechts, 4. Auflage. Boorberg, Stuttgart, 21-50.

Sumner, L. W. (2011): Assisted Death, Oxford University Press, New York.

Williams, B. (1973): The Makropoulos Case: Reflections on the Tedium of Immortality, in: Ders. (Hg.), Problems of the Self, Cambridge University Press, New York.

WitTwer, H. (2003): Selbsttötung als philosophisches Problem, Mentis, Paderborn.

WOLF, J.-C. (1993): Aktive und passive Euthanasie, Archiv für Rechts- und Sozialphilosophie 79(3), 393-415. 
Bereitgestellt von | Universitätsbibliothek Bern Angemeldet | andreas.mueller@uni-muenster.de Autorenexemplar Heruntergeladen am | 15.02.18 12:34 